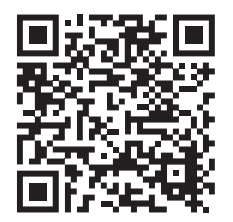

* Facultad de Estudios Superiores Zaragoza, Universidad Nacional Autónoma de México. Ciudad de México, México. ₹ Egresada de la carrera de Enfermería.

$\S$ Doctora en Enfermería.

"Comisión Nacional de Arbitraje Médico, Dirección General de Investigación y Difusión. Ciudad de México, México.

" Doctor en Psicología.

** Maestría en Ciencias de la Salud.

\# Investigador en Ciencias Médicas.

Correspondencia: $\mathrm{DCH}$, delia_jj22@hotmail.com Conflicto de intereses: Los autores declaran no tener ningún tipo de conflicto de intereses. Citar como: Chanes HD, González-Velázquez MS, Luna D, Lezana-Fernández MÁ, Meneses-González F. Manejo inadecuado de injerto en quemadura por parte del personal de enfermería en paciente pediátrico. Rev CONAMED. 2020; 25(4): 182-187. https:// dx.doi.org/10.35366/97338

Financiamiento: Ninguno.

Recibido: 02/12/2020.

Aceptado: 08/12/2020.

\section{Manejo inadecuado de injerto en quemadura por parte del personal de enfermería en paciente pediátrico}

\author{
Inadequate management of burn graft by nursing staff in pediatric patient

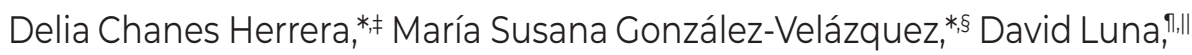

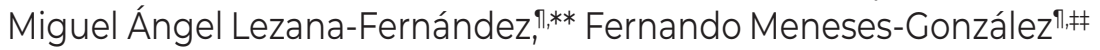

\section{RESUMEN}

Se presenta el caso de manejo inadecuado de un injerto autólogo en quemadura de un paciente pediátrico, en una unidad hospitalaria por parte del personal de enfermería. Este caso proviene del expediente desarrollado a partir de una queja presentada en la Comisión Nacional de Arbitraje Médico (CONAMED) y se enfoca en el análisis y recomendaciones sobre el cuidado enfermero en este tipo de eventos, identificando las acciones que fueron realizadas y que retardaron la recuperación del paciente.

Palabras clave: Enfermería, quemadura, injerto autólogo, pediatría.

\begin{abstract}
We present the case of inadequate management of an autologous graft in a pediatric patient's burn in a hospital unit attended by the nursing staff. This case comes from the file developed from a complaint filed with the National Medical Arbitration Commission (CONAMED) and focuses on the analysis and recommendations on nursing care in this type of event, identifying the actions that were carried out and delayed the recovery of the patient.
\end{abstract}

Keywords: Nursing, burn, autologous graft, pediatrics. 


\section{INTRODUCCIÓN}

Las quemaduras pediátricas son una de las morbilidades más frecuentes y son catalogadas como trauma prevenible. Pueden ser provocadas por agentes físicos, químicos o biológicos que afectan la piel, mucosas y el tejido subyacente. Las quemaduras pueden ser incapacitantes y graves, además de resultar en secuelas invalidantes, funcionales y estéticas, que causan trastornos psicológicos, sociales, familiares y laborales. En Estados Unidos, anualmente se atienden en unidades de emergencias por quemaduras a 120 mil menores de 20 años. En México, durante 2013, las quemaduras se ubicaron en el lugar 22 dentro de las principales causas de muerte en menores de cinco a nueve años. ${ }^{12}$

Dos tipos de pacientes pediátricos con quemadura requieren de atención hospitalaria. El primero se refiere al «niño gran quemado», el cual se diagnostica cuando el total de superficie corporal quemada es mayor de 15\%. El segundo es aquel cuya quemadura se encuentra en zonas como cara, cuello, genitales, manos, pies y articulaciones o áreas importantes de flexión.,4

El presente caso fue tomado de un expediente de la Comisión Nacional de Arbitraje Médico (CONAMED), producto de una queja médica y, por su interés clínico en la práctica de enfermería, se presenta el abordaje y análisis de una quemadura de segundo grado por escaldadura y el manejo de los cuidados brindados por el área de enfermería que llevaron al paciente a que se le realizara un doble procedimiento quirúrgico para obtener y colocar un injerto autólogo, lo cual evolucionó a una recuperación tardía y una cicatriz excavada en el área injertada. 5,6

\section{PRESENTACIÓN DEL CASO}

Femenino de 7 años de edad que acudió a una unidad de segundo nivel de atención por presentar quemaduras de segundo grado superficial en región lateral izquierda del abdomen, así como en región lateral de muslo izquierdo y quemaduras de segundo grado profundo en dorso y borde interno de pie izquierdo, con 5\% de superficie corporal quemada, motivo por el cual ameritó hospitalización a cargo de la Unidad de Quemados de ese hospital.
Sin antecedentes personales patológicos negados. Recibió como tratamiento inicial solución de Hartmann $500 \mathrm{~cm}^{3}$ cada 8 horas, dicloxacilina 500 mg intravenoso cada 6 horas, metamizol 350 mg intravenoso cada 8 horas, paracetamol 500 mg vo c/8 horas. A la paciente se le realizó una flictenolisis, curación con gasas furacinadas y vendaje almohadillado previo a su tratamiento quirúrgico.

Pasó a quirófano tres días después del ingreso hospitalario para la realización de un procedimiento de escisión tangencial y colocación de cinco aloinjertos en región lateral izquierda del abdomen. Seis días después ingresó, nuevamente a quirófano, para retiro de aloinjertos, toma de injerto cutáneo de muslo izquierdo y colocación del mismo (injerto autólogo) en el dorso del pie derecho, reportándose sin datos de infección, sin exudado, se colocaron gasas furacinadas, gasas secas, apósito especial y vendaje. No se reportaron eventualidades. Un día después egresó a cama de la Unidad de Quemados con pronóstico bueno para la vida.

Tres días posteriores a su tratamiento quirúrgi$c o$, se dio de alta al domicilio con injertos integrados, zona donadora en fase de regeneración y sin datos de proceso infeccioso. La prescripción médica fue realizar una curación diaria del área injertada o cada tercer día, de acuerdo con la evolución, cita abierta a la Unidad de Urgencias y control por su médico familiar con antibioterapia y analgesia.

La paciente acudió a cita en la Unidad Médica Familiar un día después del egreso hospitalario y fue valorada por el médico familiar, quien realizó una prescripción al servicio de curaciones con la siguiente leyenda: «...favor de realizar curación en pie izquierdo quemadura de segundo grado colocación de injerto favor de colocar gasas vaselinadas...». Con base en ello, el personal de enfermería de esa unidad realizó el procedimiento mismo que se repitió dos días después.

La paciente tuvo dos sesiones de dicho procedimiento y al acudir al servicio de curaciones para su tercera sesión, el personal de enfermería retiró el vendaje y las gasas secas que yacían sobre el injerto sin haberlas humedecido previamente, desprendiendo el injerto de forma súbita, lo que tuvo como consecuencia la pérdida del injerto y el sangrado activo del área. El personal de enfermería realizó curación con solución salina, aplicó gasa vaselinada en la herida y colocó vendaje no compresivo. 
La enfermera a cargo del procedimiento reportó posteriormente en una nota anexa al expediente que el injerto se encontraba infectado y debido a eso lo había desprendido. Sin embargo, no describió los datos de infección observados.

Un día después de este evento, la paciente fue llevada a la unidad hospitalaria donde le colocaron los injertos, realizándose valoración, reportando en nota clínica que el pie izquierdo no tenía el injerto en un $80 \%$ de la zona, sin datos de infección y fragmentos de injerto en la periferia de la lesión, además de papel microporado en la zona donadora aún sin desprenderse.

Se decidió que la paciente debía ser intervenida quirúrgicamente para una nueva obtención y colocación de autoinjerto, por lo que se le prescribió dicloxacilina y paracetamol orales; la intervención quirúrgica se realizó siete días posteriores de esa última valoración.

La paciente evolucionó con el segundo injerto favorablemente acudiendo a rehabilitación posteriormente. El diagnóstico final fue de un pie funcional con cicatriz excavada. El tratamiento desde la primera atención hasta el alta duró 119 días.

\section{DISCUSIÓN}

El manejo de las quemaduras debe ser abordado de manera multidisciplinaria con cirujanos plásticos reconstructivos, especialistas en rehabilitación, enfermería especializada, nutriólogos, infectólogos, terapia ocupacional, entre otros profesionales de la salud, en especial en quemaduras de pacientes pediátricos. ${ }^{4}$

El tratamiento de las quemaduras está enfocado en prevenir las complicaciones, entre las que destacan las infecciones secundarias en el área por ser un lugar propenso para el crecimiento de microorganismos y altamente inflamatoria. Dicho tratamiento también se enfoca en la prevención de cicatrización hipertrófica o queloide.

Las quemaduras profundas transforman la piel en escaras, siendo estas últimas las responsables de que organismos bacterianos se acumulen e invadan el tejido sano, además de propiciar la liberación de células proinflamatorias como factor de necrosis tumoral, interleucinas y citocinas, las cuales producen un estado de catabolismo que provoca el desgaste del paciente. Por lo que es de suma importancia que el tejido no viable se retire en el menor tiempo posible para disminuir la probabilidad de infección y mejorar la evolución funcional y estética, de allí que la cirugía es procedimiento electivo en el tratamiento de las quemaduras. ${ }^{4}$

Existe un tipo de cirugía llamada escisión tangencial, en la cual se reseca con dermatomos manuales por capas el tejido quemado y necrótico hasta localizar tejido viable, respetando la red linfática y dejando un mejor contorno corporal. Una vez localizado el tejido viable, el mismo queda listo para recibir un injerto de espesor parcial. Además, con este tipo de técnica se disminuyen los riesgos de infección y días de estancia hospitalaria. Con la técnica de escisión tangencial temprana se ha logrado disminuir la mortalidad, las complicaciones y mejorar la supervivencia en 50\% en adultos mayores y niños. Una de las complicaciones que se ha logrado disminuir con este procedimiento son las cicatrices hipertróficas queloides, las cuales son una de las principales disfuncionalidades que se desean evitar después de una quemadura. ${ }^{4}$

La colocación temprana de injertos se relaciona con una disminución de secuelas funcionales y cosméticas para el paciente. Mejorando con esto la calidad de vida y la prevención de problemas psicológicos y físicos.

En el presente caso se puede observar que el manejo inicial fue el adecuado, ya que se efectuó flictenolisis, se indicó terapia hídrica con solución cristaloide Ringer lactato, manejo del dolor con metamizol y paracetamol, antibioterapia con dicloxacilina para, posteriormente, realizar el manejo quirúrgico con la escisión tangencial y la colocación de injerto autólogo. Este procedimiento es consistente con el algoritmo que se muestra en la Figura 7.5 Además, los apósitos fueron retirados al cuarto día para la primera sesión de curación, acorde con los lineamientos establecidos. 6,7

La Secretaría de Salud informó que en México el 8\% de los pacientes sufren algún daño a causa de eventos adversos relacionados con la seguridad del paciente. Sin embargo, se calcula que la mayoría de este tipo pueden ser prevenibles. En relación con las ocho acciones esenciales para la seguridad del paciente, en este caso se presentó una omisión en la acción número cuatro: Seguridad en los procedimientos. Esta omisión consistió 


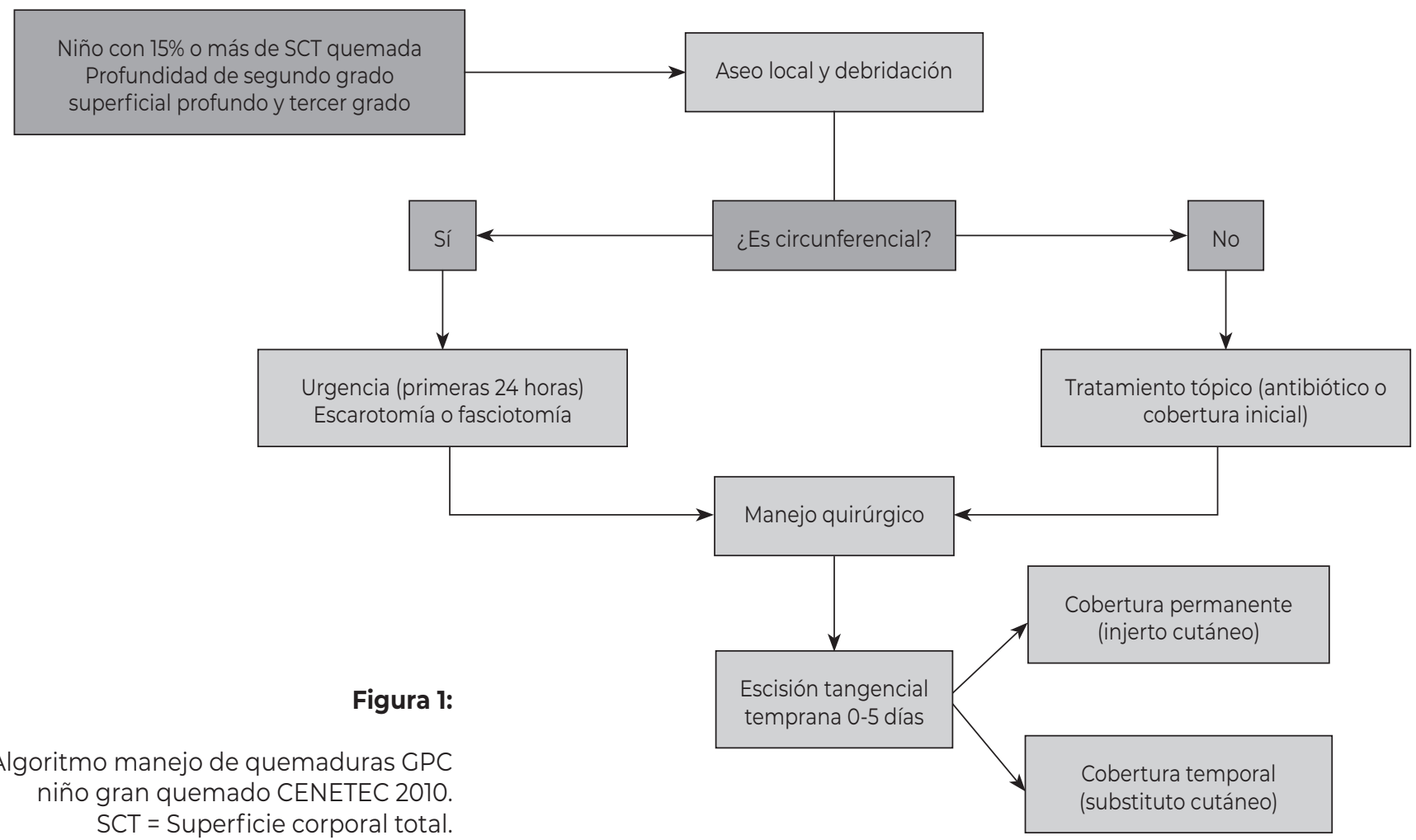

en retirar los apósitos sin dar seguimiento a las indicaciones médicas. ${ }^{8}$

La curación de los injertos en las áreas clínicas implica el retiro del vendaje con el máximo cuidado posible y despegar los apósitos colocados de forma suave, humedecidos con solución fisiológica si es necesario para evitar tirones del injerto. Una vez retirados los apósitos, se debe observar la zona, el estado del injerto y la proporción en la que se ha integrado; realizar aseo por arrastre mecánico con suero fisiológico en forma suave y secar con gasa estéril sin friccionar. ${ }^{9,10}$

Las taxonomías empleadas por enfermería como lo son la North American Nursing Diagnosis Association (NANDA)," Nursing Interventions Classification (NIC) ${ }^{12}$ y Nursing Outcomes Classification $(\mathrm{NOC})^{13}$ refieren que en el cuidado de la herida por quemadura con injerto cutáneo, el principal riesgo es el deterioro de la integridad cutánea, que se relaciona con la manipulación quirúrgica de los tejidos, por lo que el resultado esperado de la intervención de enfermería será mantener la integridad tisular. Esto será posible mediante intervenciones como la vigilancia y cuidado de la piel, en las que se observará el estado de la zona de intervención, la perfusión tisular, sequedad o humedad de la piel y evitar zonas de presión y fricción, colocando apósitos y vendajes adecuados.

Se debe valorar la zona determinando si existen datos de infección como secreción purulenta, mal olor, edema, calor, rubor y dolor. En el caso que se reporta, la enfermera mencionó que dado que el injerto se encontraba infectado lo retiró. Sin embargo, al día siguiente, el médico cirujano que valoró el área lo reportó sin datos de infección.14

Posterior a la curación se debe colocar gasas vaselinadas, gasas secas, apósito protector y vendaje. Ello tomando en cuenta la movilidad de las articulaciones, la comodidad del paciente y la expansión del posible edema. ${ }^{15}$

\section{CONCLUSIONES}

Los injertos deben ser tratados de manera especialmente cuidadosa, ya que el manejo inadecuado representa riesgos para el paciente relacionados con el retraso en la mejoría estética, riesgo de infección y costos extra para el sistema de salud. 
El cuidado adecuado de un injerto autólogo por quemadura es esencial para poder tener una evolución funcional y estética satisfactoria. Es importante que el personal de enfermería realice la lectura previa de las indicaciones médicas y que posea los conocimientos necesarios en curación de heridas. También debe tener en cuenta las acciones esenciales para la seguridad del paciente, así como los 10 correctos en enfermería, con el fin de obtener los mejores resultados tanto funcionales como estéticos y disminuir la incidencia de cicatrices queloides que representan insatisfacción e inseguridad para el paciente que las desarrolla. ${ }^{8}$

Cuando se presenta una cicatriz queloide, las taxonomías de enfermería NANDA, NIC y NOC establecen el diagnóstico de aislamiento social, durante el cual se espera mejorar la autoestima mediante una intervención de apoyo emocional, en la cual se proporciona soporte durante la negación y se anima al paciente a que exprese sus sentimientos de ira, ansiedad, tristeza, entre otros, además de proporcionarle seguridad. Todo esto con la finalidad de que la cicatriz ocasionada por la quemadura no afecte en su vida cotidiana."

Un plan de cuidados de enfermería enfocado en injerto autólogo incluye la vigilancia del cuidado de la piel y membranas mucosas. Esta intervención se conforma por las actividades necesarias que ayuden al paciente a mantener la integridad tisular, así como también mantener la buena autoestima.12,13

Por otra parte, de acuerdo con el Marco Conceptual de la Clasificación Internacional para la Seguridad del Paciente, se concluye que el presente caso se trató de un incidente en seguridad, el cual es definido como un evento o circunstancia que podría haber ocasionado u ocasionó un daño innecesario a un paciente. ${ }^{16}$

Se recomienda al personal de enfermería aplicar las acciones esenciales de seguridad del paciente antes de realizar cualquier procedimiento, mantenerse actualizado en cuanto a conocimientos clínicos generales, así como específicos del área en la que se desempeña mediante lectura continua de guías de práctica clínica y las taxonomías propias de la profesión como lo son NANDA, NIC, NOC. Todo ello con el fin de proporcionar cuidados que garanticen atención libre de riesgos y daños innecesarios al paciente, así como proporcionar un entorno seguro dentro de las instituciones de salud.

\section{AGRADECIMIENTOS}

A mis compañeros de trabajo de la Comisión Nacional de Arbitraje Médico.

\section{BiBLIOGRAFÍA}

1. Secretaría de Salud, Secretariado Técnico Consejo Nacional para la Prevención de Accidentes. Modelo para la prevención de quemaduras en grupos vulnerables en México. México, D.F.: Secretaría de Salud; 2016. Disponible en: http://conapra.salud.gob.mx/Interior/Documentos/ PACV/Modelos/ModeloQuemaduras.pdf

2. Coiffman F. Cirugía plástica, reconstructiva y estética. 2a edición. Tomo I. Barcelona: Masson-Salvat; 1994.

3. Martínez y Martínez R. Salud y enfermedad del niño y del adolescente. México, D.F.: Manual Moderno; 2013.

4. Vélez PalafoxM. Tratamiento quirúrgico de las quemaduras y protocolo de salvamento. Rev Mex Anestesiol. 2014; 37: 226-229.

5. Secretaría de Salud. Evaluación y manejo inicial de niño gran quemado, evidencias y recomendaciones. Guía de práctica clínica. México, D.F.: CENETEC; 2010. Disponible en: http://www.cenetec.salud.gob.mx/descargas/gpc/ CatalogoMaestro/448_GPC_Nixo_gran_qemado/GER Nixo_gran_quemado.pdf

6. Fernández de Cuevas GR, Hueso AT, López VZ, Martínez AS, Sánchez SS. Protocolo de curas en injerto cutáneo con Betadine ${ }^{\circledR}$ solución dérmica. D.U.E. Servicio de Cirugía Plástica. Hospital Universitario Doce de Octubre. España, 2011. Disponible en: http://www.codem.es/Adjuntos/ CODEM/Documentos/Informaciones/Publico/7e040f74Obea-427f-b327-440fe67f3617/65a09e97-9c04-4450-bd483fa4872cfd40/eb7bc8f7-557c-4069-b963-f82522743482/ Protocolo_curas_injerto_cutaneo_betadine.pdf

7. Avellaneda-Oviedo EM, Conzález-Rodríguez A, ConzálezPorto A, Palacios-Carcía P, Rodríguez-Pérez E, Bugallo SJI. Injertos en heridas. Heridas y Cicatrización. 2018; 2: 6-15.

8. Secretaría de Salud. Las acciones esenciales para la seguridad del paciente dentro del modelo de seguridad del paciente del CSC. SiNaCEAM. Ciudad de México, México. 2017. Disponible en: http://www.csg.gob.mx/descargas/pdf/ certificacion-establecimientos/modelo_de_seguridad/ acciones_ModeloCSC/AESP-ModeloSP-CSC-15.09.17.pdf

9. Secretaría de Salud. Manual clínico para la estandarización del cuidado y tratamiento a pacientes con heridas agudas y crónicas. Ciudad de México, México: Secretaría de Salud; 2016. Disponible en: http://www.cpe.salud.gob.mx/site3/ publicaciones/docs/manual_heridas.pdf 
10. Fuentes VR. Injertos cutáneos: manejo de enfermería. Ocronos. 2020; 3: 232.

11. NANDA Internacional. Diagnósticosenfermeros definiciones y clasificación 2018-2020. España: Elsevier; 2018.

12. Butcher HK, Bulecheck GM, Dochterman JM, Wagner CM. Clasificación de intervenciones de enfermería (NIC). 7a edición. España: Elsevier; 2019.

13. Moorhead S, Swanson E, Johnson M, Maas ML. Clasificación de resultados de enfermería (NOC). Medición de resultados en salud. 6a edición. España: Elsevier; 2019.

14. European Wound Management Association (EWMA). Position document: identifying criteria for wound infection. London: MEP Ltd; 2005. Disponible en: https:// www.ulceras.net/publicaciones/Identificacion\%20de\%20 los\%20criterios\%20de\%20infeccion.pdf

15. Gallardo GR, Ruiz PJG, Torres PRM, Díaz OJ. Estado actual del manejo urgente de las quemaduras (II). Conducta a seguir ante un paciente quemado. Emergencias. 2001; 13: 188-196.

16. Organización Mundial de la Salud. Marco conceptual de clasificación para la seguridad del paciente. Organización Mundial de la Salud. Disponible en: https://www.who.int/patientsafety/implementation/icps/ icps_full_report_es.pdf 